OPEN ACCESS

Edited by: Diego Ruano,

Universidad de Sevilla, Spain

Reviewed by: Alessandro Martorana Università degli Studi di Roma Tor Vergata, Italy Gessica Sala,

Università degli Studi di Milano Bicocca, Italy

*Correspondence: David A. Loeffler david.loeffler@beaumont.org

Received: 02 March 2018 Accepted: 17 April 2018

Published: 08 May 2018

Citation: Loeffler DA, Klaver AC, Coffey MP and Aasly JO (2018) Cerebrospinal Fluid Concentration of Key Autophagy Protein Lamp2 Changes Little During Normal Aging. Front. Aging Neurosci. 10:130. doi: 10.3389/fnagi.2018.00130

\section{Cerebrospinal Fluid Concentration of Key Autophagy Protein Lamp2 Changes Little During Normal Aging}

\author{
David A. Loeffler ${ }^{1 *}$, Andrea C. Klaver ${ }^{1}$, Mary P. Coffey ${ }^{2}$ and Jan O. Aasly ${ }^{3}$ \\ ${ }^{1}$ Department of Neurology, Beaumont Hospital-Royal Oak, Beaumont Health, Royal Oak, MI, United States, ${ }^{2}$ Department \\ of Biostatistics, Beaumont Hospital-Royal Oak, Beaumont Health, Royal Oak, MI, United States, ${ }^{3}$ Department of Neurology, \\ St. Olav's Hospital, Trondheim, Norway
}

Autophagy removes both functional and damaged intracellular macromolecules from cells via lysosomal degradation. Three autophagic mechanisms, namely macroautophagy, chaperone-mediated autophagy (CMA), and microautophagy, have been described in mammals. Studies in experimental systems have found macroautophagy and CMA to decrease with normal aging, despite the fact that oxidative stress, which can activate both processes, increases with normal aging. Whether autophagic mechanisms decrease in the human brain during normal aging is unclear. The primary objective of this study was to examine the association of a major autophagy protein, lysosome-associated membrane glycoprotein (lamp2), with age in cerebrospinal fluid (CSF) samples from healthy subjects. Lamp2 consists of three isoforms, lamp2a, 2b and 2c, all of which participate in autophagy. Lamp2's CSF concentration decreases in Parkinson's disease (PD) and increases in Alzheimer's disease (AD), but whether its CSF concentration changes during normal aging has not been investigated. Our secondary objectives were to examine the associations of lamp2's CSF concentration with CSF levels of the molecular chaperone heat shock 70-kDa protein (HSPA8), which interacts with lamp2a in CMA, and oxidative stress markers 8-hydroxy-2'-deoxyguanosine (8-OHdG), 8-isoprostane (8-ISO) and Total Antioxidant Capacity (TAC) in healthy subjects. We found lamp2's observed associations with these variables to be weak, with all Kendall's tau-b absolute values $\leq 0.20$. These results suggest that CSF lamp2 concentration changes little during normal aging and does not appear to be associated with HSPA8 or oxidative stress. Further studies are indicated to determine the relationship between CSF lamp2 concentration and brain autophagic processes.

Keywords: aging, autophagy, CSF, HSPA8, lamp2, oxidative stress

\section{INTRODUCTION}

Proteostasis involves regulation of protein transcription, translation, folding, trafficking, processing, assembly/disassembly, localization, and degradation (Douglas and Dillin, 2010). Cells attempt to maintain proteostasis via the autophagy-lysosomal pathway and the ubiquitin-proteasome system (Xilouri and Stefanis, 2016). Three autophagic processes, namely 
macroautophagy, chaperone-mediated autophagy (CMA) and microautophagy have been described in mammals (Cuervo, 2004). Based on studies in rat livers and human fibroblast cultures, macroautophagy and CMA are thought to decrease with normal aging (Cuervo and Dice, 2000; Del Roso et al., 2003). If these processes decrease in the brain during normal aging, this could contribute to aging being a primary risk factor for the two most prevalent neurodegenerative disorders, Alzheimer's disease (AD) and Parkinson's disease (PD; Jeppesen et al., 2011; Xilouri and Stefanis, 2016). The few investigations of age-related changes in brain autophagy have produced conflicting results. Genome-wide analyses of human brain specimens found downregulation of a key macroautophagy protein, beclin-1 (Shibata et al., 2006), and of major autophagy genes including Atg5 and Atg7 (Lipinski et al., 2010b); the latter study also found upregulation of genes involved in regulation and mediation of the mitogen-activated protein (MAP) kinase pathway, which was predicted in an earlier study (Lipinski et al., 2010a) to result in suppression of autophagy. Conversely, a proteomics study of human cerebrospinal fluid (CSF) found that three proteins with the gene ontology classification of "autophagy" namely myoglobin, MMP8 and HMW kininogen (none of which is a major autophagy protein), increased with age (Baird et al., 2012). An age-related increase in macroautophagy in the rat brain has also been reported (Gamerdinger et al., 2009).

There are no established biomarkers in cerebrospinal fluid (CSF) for monitoring brain autophagy. Beclin-1, p62 and LC3-II have been suggested as macroautophagy biomarkers (Karim et al., 2007; Pattingre et al., 2008; Li et al., 2015; Au et al., 2017) but, to our knowledge, age-related changes in these proteins have not been examined in CSF. We recently reported (Loeffler et al., 2016) that the CSF concentration of heat shock $70-\mathrm{kDa}$ protein (HSPA8, also known as hsc70 and hsc73), a molecular chaperone involved in CMA, decreases with aging, but whether changes in CSF HSPA8 levels reflect changes in brain CMA is unknown. In the present study we explored changes in the CSF concentration of another autophagy protein, lysosome-associated membrane glycoprotein 2 (lamp2), during normal aging. Lamp2 has three isoforms, lamp2a, $2 \mathrm{~b}$ and $2 \mathrm{c}$, which are generated by alternative splicing of the LAMP2 gene and differ in their C-terminus sequences (Gough et al., 1995). All three isoforms participate in autophagy; lamp2a's binding by the substrate protein-heat shock $70-\mathrm{kDa}$ protein (HSPA8) complex is rate-limiting for CMA (Cuervo and Dice, 1996), lamp2b is involved in macroautophagy (and may be required for autophagosome-lysosome fusion; Nishino et al., 2000; Rowland et al., 2016), and lamp2c is a receptor for autophagic degradation of DNA and RNA (Fujiwara et al., 2015). Lamp2's CSF concentration has been reported to decrease in PD (Boman et al., 2016; Klaver et al., 2018) and to increase in $\mathrm{AD}$ (Armstrong et al., 2014), in accordance with reports of decreased lamp2a and HSPA8 (referred to in that study as hsc70) in PD brain specimens (Alvarez-Erviti et al., 2010) and increased transcription of positive regulatory genes for autophagy in AD brain specimens (Lipinski et al., 2010b). Lamp2's gene expression in human leukocytes decreases with normal aging (Huang et al., 2012), but whether its CSF concentration changes during normal aging is unknown. Our primary objective in this study was to examine this issue. Our secondary objectives were to examine lamp2's correlations, in CSF from healthy subjects, with HSPA8 and with markers of oxidative stress (8-hydroxy-2'-deoxyguanosine [8-OHdG], 8-isoprostane [8-ISO], and Total Antioxidant Capacity [TAC]). TAC includes nonenzymatic low molecular weight antioxidants such as ascorbic acid and glutathione (Alho et al., 1998; Bartosz, 2003); because a decrease in TAC could result in elevated oxidative stress, it may be an indirect marker for oxidative stress (Mandrioli et al., 2006). Oxidative stress increases in the aged brain (Navarro et al., 2002; Vanguilder and Freeman, 2011) and can activate both CMA and macroautophagy (Kiffin et al., 2004; Kaushik et al., 2010). In a previous study with these samples (Loeffler et al., 2016), we found the CSF concentration of the oxidative stress marker $8-\mathrm{OHdG}$ to increase with age (Spearman rho $=0.61$.

\section{MATERIALS AND METHODS}

\section{Study Subjects}

Details of the subjects whose CSF samples were used in this study were reported previously (Loeffler et al., 2016). The subjects were recruited by neurologist Jan Aasly, at St. Olav's Hospital, Trondheim, Norway. They were tested for and lacked known PD-related mutations in the LRRK2, PARK2, PARK7, PINK1 and SNCA genes, and had no detectable cognitive or neurological impairments. All procedures involving the study subjects, including obtaining of written informed consent, were performed at St. Olav's Hospital in accordance with the Declaration of Helsinki of 1975 and its subsequent amendments. The study was approved by the Regional Committee for Medical Research Ethics, Central Norway, for the procedures performed at St. Olav's Hospital. Lumbar CSF samples were obtained using Parkinson's Progression Markers Initiative (PPMI) biospecimen collection procedures (Parkinson's Progression Markers Initiative, 2014). The study was given exempt status from the Institutional Review Board of Beaumont Health (Royal Oak, MI, USA) for the procedures performed in the Neurology Research Laboratory at Beaumont Hospital-Royal Oak (MI, USA), where lamp2 was measured in de-identified CSF samples.

\section{Lamp2 Measurements}

Lamp2 was measured using the ELISA Kit for Lysosomal Associated Membrane Protein 2 (LAMP2; cat. \# SEB464Hu) from Cloud-Clone Corp. (Katy, TX, USA). The lower limit of detection for lamp2 in the ELISA kit was stated by the manufacturer to be $26.2 \mathrm{pg} / \mathrm{mL}$. The standard curve in the assay ranged from $62.5 \mathrm{pg} / \mathrm{mL}$ to $4000 \mathrm{pg} / \mathrm{mL}$. The lamp2 concentration of each sample was measured in duplicate after diluting the sample with an equal volume of $0.01 \mathrm{M}$ PBS buffer, $\mathrm{pH}$ 7.2; the lamp2 concentrations used in statistical analyses were the means of these duplicate measurements. The standard curve was generated using Softmax 
Pro software (version 3.0; Molecular Devices Corp., Sunnyvale, CA, USA) using the log-log function as recommended by the manufacturer, and the concentration of lamp2 in each sample was calculated by Softmax. One sample produced a mean optical density value which was below the value for the lowest point on the standard curve; for statistical purposes, the sample was assigned a concentration of $26.2 \mathrm{pg} / \mathrm{mL}$, the lower limit of sensitivity for the assay. After accounting for the dilution factor, the concentration of the sample was listed as $52.4 \mathrm{pg} / \mathrm{mL}$.

\section{Statistics}

Measures were summarized with means \pm SDs for variables which were normally distributed, and with medians and ranges for variables which were not normally distributed. Lamp2 values were distributed abnormally (strongly skewed right with extreme outliers); natural-log transformation produced a reasonably normal distribution. Kendall's rank-correlation coefficient (Kendall's tau-b) with associated 95\% confidence intervals (CI), and scatterplots with best-fit regression lines and locally weighted scatterplot smoother (LOWESS) curves were used to examine lamp2's associations with age, HSPA8, 8-OHdG, 8 -ISO and TAC. Differences for lamp2 concentrations between male and female subjects were examined with $t$-tests. Original (non-transformed) lamp2 values were used for Kendall's tau-b calculations, while log-transformed lamp2 values were used for scatterplots and gender comparisons. Statistical analysis used The SAS System for Windows version 9.3 (SAS Institute Inc., Cary, NC, USA) and Minitab Release 14 (Minitab Inc., State College, PA, USA) was used for graphs.

\section{RESULTS}

\section{Associations of CSF Lamp2 Concentration With Other Variables}

Numeric summaries for age and lamp2, HSPA8, 8-OHdG, 8-ISO, and TAC concentrations are shown in Table 1, and lamp2's associations with the other variables are shown in Table 2. Lamp2 was poorly correlated with age (Kendall's tau- $b=0.16$; $95 \%$ CI for tau-b $=[-0.08,0.40])$ and with the other variables (all Kendall's tau-b absolute values $\leq 0.20$ ). The 95\% CIs for lamp2's associations with age $[-0.08,0.40]$ and with 8 -OHdG $[-0.01,0.41]$ do not rule out the possibility of moderate positive associations.

The scatterplot of log-transformed lamp2 vs. age (Figure 1) indicated that the LOWESS curve closely followed the regression line, suggesting that the weak positive relationship that we observed between lamp2 and age was no more complex than linear. Conversely, in the scatterplot for log-transformed lamp2 vs. 8-OHdG (Figure 2), the LOWESS curve was steeper than the regression line to an $8-\mathrm{OHdG}$ concentration of approximately $900 \mathrm{pg} / \mathrm{mL}$, suggesting that lamp2 might increase with 8-OHdG to this 8-OHdG concentration, then level off or decrease. Scatterplots of log-transformed lamp2 vs. 8-ISO,
TABLE 1 | Age and cerebrospinal fluid (CSF) lysosome-associated membrane glycoprotein 2 (lamp2), heat shock 70-kDa protein (HSPA8), 8-OHdG, 8-ISO and TAC concentrations in study subjects.

Variable

Summary measure

Age (years) - median, range

lamp2 (pg/mL) - median, range

$\mathrm{HSPA} 8(\mathrm{ng} / \mathrm{mL})-$ mean $\pm \mathrm{SD}$

8-OHdG $(\mathrm{pg} / \mathrm{mL})$ - mean $\pm \mathrm{SD}$

8-ISO (pg/mL) - median, range

TAC (mM) - median, range

$55.5(20-75)$

$350.8(52.4-10,561)$

$0.5 \pm 0.2$

$811.3 \pm 160.8$

$6.5(3.2-16.7)$

$0.4(0.2-0.6)$

Study subjects were 18 males and 16 females. The data for HSPA8, 8-OHdG, 8-ISO and TAC were taken from our previous study with these samples (Loeffler et al., 2016). 8-OHdG, 8-hydroxy-2/-deoxyguanosine; 8-ISO, 8-isoprostane; TAC, Total Antioxidant Capacity.

TABLE 2 | Kendall's rank-correlation coefficients of CSF lamp2 concentrations with age, HSPA8 and oxidative stress measures in study subjects.

\begin{tabular}{lcc}
\hline Variable & Kendall's Tau-b & $\mathbf{9 5 \%} \mathbf{C l}$ for Kendall's Tau-b \\
\hline Age & 0.16 & $(-0.08,0.40)$ \\
HSPA8 & -0.15 & $(-0.39,0.09)$ \\
8-OHdG & 0.20 & $(-0.01,0.41)$ \\
8-ISO & -0.13 & $(-0.33,0.07)$ \\
TAC & 0.09 & $(-0.17,0.34)$ \\
\hline
\end{tabular}

CSF lamp2 concentration was weakly correlated with the other variables. HSPA8, heat shock 70-kDa protein 8; 8-OHdG, 8-hydroxy-2'-deoxyguanosine; 8-ISO, 8-isoprostane; TAC, Total Antioxidant Capacity.

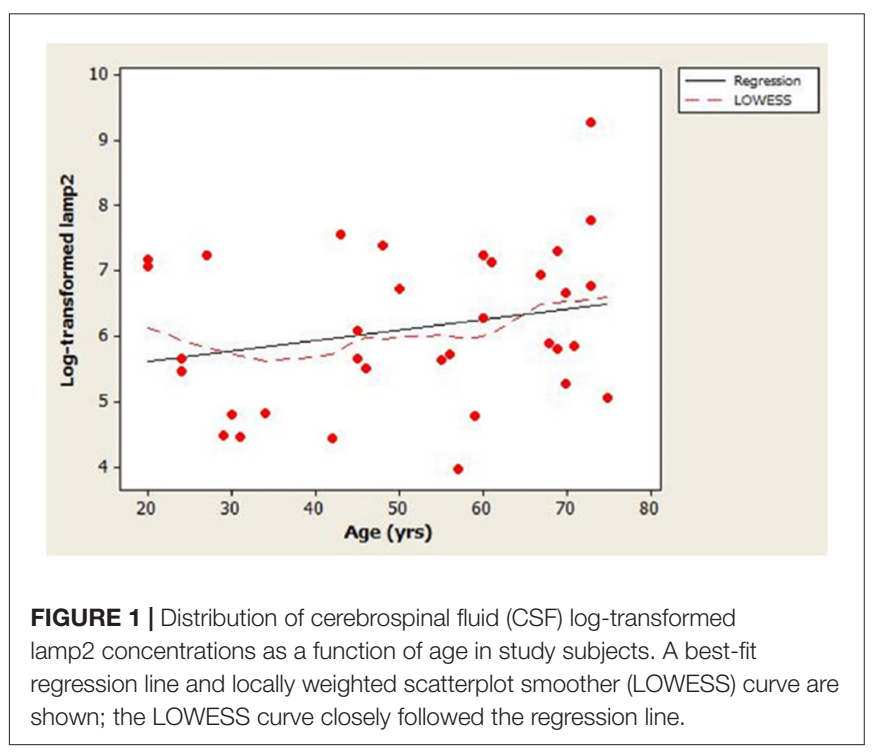

TAC, and HSPA8 (not shown) did not suggest an association of lamp2 with any of these variables.

\section{Gender Differences in CSF Lamp2 Concentration}

No significant differences were found for CSF lamp2 concentration between genders $(p=0.80)$. Because the male study subjects tended to be younger than the female study subjects, the gender comparison was limited to subjects 


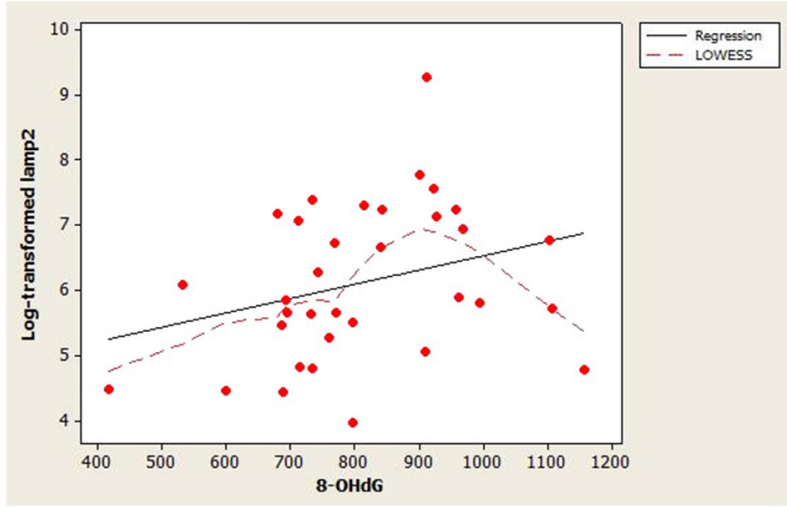

FIGURE 2 | Distribution of CSF log-transformed lamp2 concentrations as a function of 8-hydroxy-2'-deoxyguanosine (8-OHdG) concentrations in study subjects. A best-fit regression line and LOWESS curve are shown. The LOWESS curve suggested that log-transformed lamp2 may increase with 8-OHdG to approximately $900 \mathrm{pg} / \mathrm{mL}$; after that point it may level off or decrease.

$\geq 40$ years old, namely 16 females (mean age 60.3) and nine males (mean age 60.1).

\section{DISCUSSION}

The main finding in this study was that the association between CSF lamp2 concentration and age appeared to be weak in healthy subjects. The scatter plot for the distribution of log-transformed lamp2 vs. age (Figure 1) indicated large variability in lamp2 concentrations among similar-aged individuals. Age could explain, at best, only a small proportion of this variability (adjusted $R^{2}=1.4 \%$ ). Whether the lack of age-related changes in CSF lamp2 reflects a similar lack of change in brain lamp2 and/or brain autophagic processes during normal aging is unknown; such a determination would require measurement of lamp2 on lysosomal membranes, as well as assessment of CMA and macroautophagy activities, in normal brain specimens across a wide age range.

Because the concentration of lamp2 is the sum of the concentrations of all three lamp2 isoforms, the lack of change that we found in CSF lamp2 concentration during normal aging does not necessarily indicate a lack of change in the concentrations of its isoforms. A study measuring mRNA for lamp2's isoforms in normal human anterior cingulate and occipital cortex (Murphy et al., 2015) found that only $5 \%$ of lamp2 mRNA encoded for lamp2a, whereas $85 \%$ encoded for lamp2c. Whether lamp2's isoforms are similarly distributed in CSF is unknown. If lamp2a accounts for only $5 \%$ of lamp2's CSF concentration, then it may be difficult to detect by standard techniques such as ELISA or western blot. No commercial ELISAs are available for measuring lamp2's isoforms.

The correlation between lamp2 and HSPA8 was -0.15 . It is unclear if a stronger association between these proteins should have been expected. Activation of CMA is often mediated by increases in the lysosomal levels of both lamp2a and HSPA8 (Agarraberes et al., 1997), but as discussed above, changes in lamp2's CSF concentration may not reflect those of lamp2a. HSPA8 is the main housekeeping member of the heat shock protein 70 family and is involved in many other processes in addition to CMA (Stricher et al., 2013), so its concentration in CSF may not reflect brain CMA activity.

Lamp2's correlations with the oxidative stress markers were also weak. This suggests that lamp2 CSF concentration in healthy individuals may not be associated with, and may not be influenced by, oxidative stress. Although the LOWESS curve in Figure 2 suggested that lamp2 might increase with 8-OHdG to approximately $900 \mathrm{pg} / \mathrm{mL} 8-\mathrm{OHdG}$, this finding requires confirmation.

The number of subjects in this study was sufficient for investigating lamp2's associations with age, HSPA8, and oxidative stress markers. Although the CI for lamp2's associations with age and $8-\mathrm{OHdG}$ do not rule out the possibility of moderate positive associations, they suggest that negative associations between these variables are unlikely. This was a cross-sectional study which measured CSF lamp2 at a single age in each subject; a study measuring each subject's CSF lamp2 at multiple time points (e.g., over multiple decades) might have increased our ability to detect age-related changes in lamp2.

Recent studies have indicated a relationship between autophagic processes and lipid metabolism. In experimental models, lipid loading exerts inhibitory effects on both macroautophagy and CMA in hepatocytes (Koga et al., 2010; Rodriguez-Navarro et al., 2012). In the latter study, the inhibitory effects of lipid challenge on CMA were ascribed to decreased stability of lamp2a at lysosomal membranes, which resulted in lowering of its concentration there. Whether increased lipid intake similarly causes changes in the lamp2a and/or total lamp2 concentrations in the CNS is unknown. All of our subjects had similar basal metabolic index classifications, and we were not aware of differences in their eating habits. However, because the basal metabolic rate decreases each decade after age 20 by $1 \%-2 \%$ (Manini, 2010), we cannot rule out the possibility that this could have influenced the correlation (which was weak) that we found between CSF lamp2 and subject age.

We conclude that CSF lamp2 concentration appears to change little during normal aging, and it appears to be poorly associated with CSF concentrations of HSPA8 and of oxidative stress markers. Further studies are indicated to clarify the effects of aging on autophagic processes in the human brain and to identify CSF biomarkers for these processes.

\section{AUTHOR CONTRIBUTIONS}

DAL directed the study and prepared the original and revised manuscript. ACK performed the assays, collated the data and reviewed the manuscript. MPC performed the statistical analysis and assisted with manuscript revisions. JOA recruited the patients, collected the CSF samples and reviewed the manuscript. 


\section{FUNDING}

This study was supported by a generous donation from Ms. Marilyn Bishop to the Beaumont Foundation.

\section{REFERENCES}

Agarraberes, F. A., Terlecky, S. R., and Dice, J. F. (1997). An intralysosomal hsp70 is required for a selective pathway of lysosomal protein degradation. J. Cell Biol. 137, 825-834. doi: 10.1083/jcb.137.4.825

Alho, H., Leinonen, J. S., Erhola, M., Lönnrot, K., and Aejmelaeus, R. (1998). Assay of antioxidant capacity of human plasma and CSF in aging and disease. Restor. Neurol. Neurosci. 12, 159-165.

Alvarez-Erviti, L., Rodriguez-Oroz, M. C., Cooper, J. M., Caballero, C., Ferrer, I., Obeso, J. A., et al. (2010). Chaperone-mediated autophagy markers in Parkinson disease brains. Arch. Neurol. 67, 1464-1472. doi: 10.1001/archneurol.2010.198

Armstrong, A., Mattsson, N., Appelqvist, H., Janefjord, C., Sandin, L., Agholme, L., et al. (2014). Lysosomal network proteins as potential novel CSF biomarkers for Alzheimer's disease. Neuromolecular Med. 16, 150-160. doi: 10.1007/s12017013-8269-3

Au, A. K., Aneja, R. K., Bayır, H., Bell, M. J., Janesko-Feldman, K., Kochanek, P. M., et al. (2017). Autophagy biomarkers beclin 1 and p62 are increased in cerebrospinal fluid after traumatic brain injury. Neurocrit. Care 26, 348-355. doi: $10.1007 / \mathrm{s} 12028-016-0351-\mathrm{x}$

Baird, G. S., Nelson, S. K., Keeney, T. R., Stewart, A., Williams, S., Kraemer, S., et al. (2012). Age-dependent changes in the cerebrospinal fluid proteome by slow off-rate modified aptamer array. Am. J. Pathol. 180, 446-456. doi: 10.1016/j. ajpath.2011.10.024

Bartosz, G. (2003). Total antioxidant capacity. Adv. Clin. Chem. 37, 219-292. doi: 10.1016/S0065-2423(03)37010-6

Boman, A., Svensson, S., Boxer, A., Rojas, J. C., Seeley, W. W., Karydas, A., et al. (2016). Distinct lysosomal network protein profiles in Parkinsonian syndrome cerebrospinal fluid. J. Parkinsons Dis. 6, 307-315. doi: 10.3233/JPD-150759

Cuervo, A. M. (2004). Autophagy: in sickness and in health. Trends Cell Biol. 14, 70-77. doi: 10.1016/j.tcb.2003.12.002

Cuervo, A. M., and Dice, J. F. (1996). A receptor for the selective uptake and degradation of proteins by lysosomes. Science 273, 501-503. doi: 10.1126/science.273.5274.501

Cuervo, A. M., and Dice, J. F. (2000). Age-related decline in chaperone-mediated autophagy. J. Biol. Chem. 275, 31505-31513. doi: 10.1074/jbc.M002102200

Del Roso, A., Vittorini, S., Cavallini, G., Donati, A., Gori, Z., Masini, M., et al. (2003). Ageing-related changes in the in vivo function of rat liver macroautophagy and proteolysis. Exp. Gerontol. 38, 519-527. doi: 10.1016/s0531-5565(03)00002-0

Douglas, P. M., and Dillin, A. (2010). Protein homeostasis and aging in neurodegeneration. J. Cell. Biol. 190, 719-729. doi: 10.1083/jcb.201005144

Fujiwara, Y., Hase, K., Wada, K., and Kabuta, T. (2015). An RNautophagy/DNautophagy receptor, LAMP2C, possesses an arginine-rich motif that mediates RNA/DNA-binding. Biochem. Biophys. Res. Commun. 460, 281-286. doi: 10.1016/j.bbrc.2015.03.025

Gamerdinger, M., Hajieva, P., Kaya, A. M., Wolfrum, U., Hartl, F. U., Behl, C., et al. (2009). Protein quality control during aging involves recruitment of the macroautophagy pathway by BAG3. EMBO J. 28, 889-901. doi: 10.1038/emboj. 2009.29

Gough, N. R., Hatem, C. L., and Fambrough, D. M. (1995). The family of LAMP-2 proteins arises by alternative splicing from a single gene: characterization of the avian LAMP-2 gene and identification of mammalian homologs of LAMP-2b and LAMP-2c. DNA Cell Biol. 14, 863-867. doi: 10.1089/dna.1995.14.863

Huang, J., Xu, J., Pang, S., Bai, B., and Yan, B. (2012). Age-related decrease of the LAMP-2 gene expression in human leukocytes. Clin. Biochem. 45, 1229-1232. doi: 10.1016/j.clinbiochem.2012.06.016

Jeppesen, D. K., Bohr, V. A., and Stevnsner, T. (2011). DNA repair deficiency in neurodegeneration. Prog. Neurobiol. 94, 166-200. doi: 10.1016/j.pneurobio. 2011.04.013

Karim, M. R., Kanazawa, T., Daigaku, Y., Fujimura, S., Miotto, G., and Kadowaki, M. (2007). Cytosolic LC3 ratio as a sensitive index of

\section{ACKNOWLEDGMENTS}

We thank the individuals who participated in this study and their families.

macroautophagy in isolated rat hepatocytes and H4-II-E cells. Autophagy 3, 553-560. doi: 10.4161/auto.4615

Kaushik, S., Singh, R., and Cuervo, A. M. (2010). Autophagic pathways and metabolic stress. Diabetes Obes. Metab. 12, 4-14. doi: 10.1111/j.1463-1326. 2010.01263.x

Kiffin, R., Christian, C., Knecht, E., and Cuervo, A. M. (2004). Activation of chaperone-mediated autophagy during oxidative stress. Mol. Biol. Cell 15, 4829-4840. doi: 10.1091/mbc.e04-06-0477

Klaver, A. C., Coffey, M. P., Aasly, J. O., and Loeffler, D. A. (2018). CSF lamp2 concentrations are decreased in female Parkinson's disease patients with LRRK2 mutations. Brain Res. 1683, 12-16. doi: 10.1016/j.brainres.2018. 01.016

Koga, H., Kaushik, S., and Cuervo, A. M. (2010). Inhibitory effect of intracellular lipid load on macroautophagy. Autophagy 6, 825-827. doi: 10.1096/fj.09144519

Li, H., Qiu, S., Li, X., Li, M., and Peng, Y. (2015). Autophagy biomarkers in CSF correlates with infarct size, clinical severity and neurological outcome in AIS patients. J. Transl. Med. 13:359. doi: 10.1186/s12967-015-0726-3

Lipinski, M. M., Hoffman, G., Ng, A., Zhou, W., Py, B. F., Hsu, E., et al. (2010a). A genome-wide siRNA screen reveals multiple mTORC1 independent signaling pathways regulating autophagy under normal nutritional conditions. Dev. Cell 18, 1041-1052. doi: 10.1016/j.devcel.2010.05.005

Lipinski, M. M., Zheng, B., Lu, T., Yan, Z., Py, B. F., Ng, A., et al. (2010b). Genomewide analysis reveals mechanisms modulating autophagy in normal brain aging and in Alzheimer's disease. Proc. Natl. Acad. Sci. U S A 107, 14164-14169. doi: 10.1073/pnas.1009485107

Loeffler, D. A., Klaver, A. C., Coffey, M. P., Aasly, J. O., and LeWitt, P. A. (2016). Age-related decrease in heat shock 70-kDa protein 8 in cerebrospinal fluid is associated with increased oxidative stress. Front. Aging Neurosci. 8:178. doi: 10.3389/fnagi.2016.00178

Mandrioli, J., Del Rio, D., Zini, A., Nichelli, P., Merelli, E., Beltrami, D., et al. (2006). Total antioxidant capacity of cerebrospinal fluid is decreased in patients with motor neuron disease. Neurosci. Lett. 401, 203-208. doi: 10.1016/j.neulet. 2006.03.013

Manini, T. M. (2010). Energy expenditure and aging. Ageing Res. Rev. 9, 1-11. doi: 10.1016/j.arr.2009.08.002

Murphy, K. E., Gysbers, A. M., Abbott, S. K., Spiro, A. S., Furuta, A., Cooper, A., et al. (2015). Lysosomal-associated membrane protein 2 isoforms are differentially affected in early Parkinson's disease. Mov. Disord. 30, 1639-1647. doi: 10.1002/mds.26141

Navarro, A., Sánchez Del Pino, M. J., Gómez, C., Peralta, J. L., and Boveris, A. (2002). Behavioral dysfunction, brain oxidative stress and impaired mitochondrial electron transfer in aging mice. Am. J. Physiol. Regul. Integr. Comp. Physiol. 282, R985-R992. doi: 10.1152/ajpregu.00537.2001

Nishino, I., Fu, J., Tanji, K., Yamada, T., Shimojo, S., Koori, T., et al. (2000). Primary LAMP-2 deficiency causes X-linked vacuolar cardiomyopathy and myopathy (Danon disease). Nature 406, 906-910. doi: 10.1038/350 22604

Parkinson's Progression Markers Initiative. (2014). Biospecimen collection, processing, and shipment manual. Available online at: http://www.ppmi-info. org/wp-content/uploads/2014/12/PPMI-Biologics-Manual-12-23-14.pdf

Pattingre, S., Espert, L., Biard-Piechaczyk, M., and Codogno, P. (2008). Regulation of macroautophagy by mTOR and Beclin 1 complexes. Biochimie 90, 313-323. doi: 10.1016/j.biochi.2007.08.014

Rodriguez-Navarro, J. A., Kaushik, S., Koga, H., Dall'Armi, C., Shui, G., Wenk, M. R., et al. (2012). Inhibitory effect of dietary lipids on chaperonemediated autophagy. Proc. Natl. Acad. Sci. U S A 109, E705-E714. doi: 10.1073/pnas.1113036109

Rowland, T. J., Sweet, M. E., Mestroni, L., and Taylor, M. R. G. (2016). Danon disease-dysregulation of autophagy in a multisystem disorder with cardiomyopathy. J. Cell Sci. 129, 2135-2143. doi: 10.1242/jcs. 184770 
Shibata, M., Lu, T., Furuya, T., Degterev, A., Mizushima, N., Yoshimori, T., et al. (2006). Regulation of intracellular accumulation of mutant Huntingtin by Beclin 1. J. Biol. Chem. 281, 14474-14485. doi: 10.1074/jbc.M600364200

Stricher, F., Macri, C., Ruff, M., and Muller, S. (2013). HSPA8/HSC70 chaperone protein: structure, function, and chemical targeting. Autophagy 9, 1937-1954. doi: 10.4161 /auto. 26448

Vanguilder, H. D., and Freeman, W. M. (2011). The hippocampal neuroproteome with aging and cognitive decline: past progress and future directions. Front. Aging Neurosci. 3:8. doi: 10.3389/fnagi.2011.00008

Xilouri, M., and Stefanis, L. (2016). Chaperone mediated autophagy in aging: starve to prosper. Ageing Res. Rev. 32, 13-21. doi: 10.1016/j.arr.2016.07.001
Conflict of Interest Statement: The authors declare that the research was conducted in the absence of any commercial or financial relationships that could be construed as a potential conflict of interest.

Copyright (c) 2018 Loeffler, Klaver, Coffey and Aasly. This is an open-access article distributed under the terms of the Creative Commons Attribution License (CC BY). The use, distribution or reproduction in other forums is permitted, provided the original author(s) and the copyright owner are credited and that the original publication in this journal is cited, in accordance with accepted academic practice. No use, distribution or reproduction is permitted which does not comply with these terms. 